\title{
The cluster Abell 780: an optical view ${ }^{\star}$
}

\author{
F. Durret ${ }^{1,2}$, E. Slezak ${ }^{3}$, and C. Adami ${ }^{4}$ \\ 1 UPMC Université Paris 06, UMR 7095, Institut d'Astrophysique de Paris, 75014 Paris, France \\ e-mail: durret@iap.fr \\ 2 CNRS, UMR 7095, Institut d'Astrophysique de Paris, 75014 Paris, France \\ 3 University of Nice Sophia Antipolis, CNRS, Observatoire de la Côte d'Azur, BP 4229, 06304 Nice Cedex 4, France \\ ${ }^{4}$ LAM, Pôle de l'Étoile Site de Château-Gombert, 38 rue Frédéric Joliot-Curie, 13388 Marseille Cedex 13, France
}

Received 3 April 2009 / Accepted 29 July 2009

\section{ABSTRACT}

\begin{abstract}
Context. The Abell 780 cluster, better known as the Hydra A cluster, has been thouroughly analyzed in X-rays. However, little is known about its optical properties.

Aims. We propose to derive the galaxy luminosity function (GLF) in this apparently relaxed cluster and to search for possible environmental effects by comparing the GLFs in various regions and by looking at the galaxy distribution at large scale around Abell 780 . Methods. Our study is based on optical images obtained with the ESO $2.2 \mathrm{~m}$ telescope and WFI camera in the $B$ and $R$ bands, covering a total region of $67.22 \times 32.94 \mathrm{arcmin}^{2}$, or $4.235 \times 2.075 \mathrm{Mpc}^{2}$ for a cluster redshift of 0.0539 .

Results. In a region of $500 \mathrm{kpc}$ radius around the cluster center, the GLF in the $R$ band shows a double structure, with a broad and flat bright part and a flat faint end that can be fit by a power law with an index $\alpha \sim-0.85 \pm 0.12$ in the $20.25 \leq R \leq 21.75$ interval. If we divide this $500 \mathrm{kpc}$ radius region in north+south or east+west halves, we find no clear difference between the GLFs in these smaller regions. No obvious large-scale structure is apparent within $5 \mathrm{Mpc}$ from the cluster, based on galaxy redshifts and magnitudes collected from the NED database in a much larger region than that covered by our data, suggesting that there is no major infall of material in any preferential direction. However, the Serna-Gerbal method reveals a gravitationally bound structure of 27 galaxies, which includes the $\mathrm{cD}$, and of a more strongly gravitationally bound structure of 14 galaxies.

Conclusions. These optical results agree with the overall relaxed structure of Abell 780 previously derived from X-ray analyses.
\end{abstract}

Key words. galaxies: clusters: individual: Abell 780 - galaxies: luminosity function, mass function

\section{Introduction}

Galaxy evolution is known to be influenced by environmental effects, which are particularly strong in galaxy clusters, where the effects of several physical processes such as ram pressure stripping, galaxy harassment, or infall of field galaxies are commonly observed. The analysis of galaxy luminosity functions (GLF) and, in particular, of their faint-end slopes in several wavebands, is a good way to trace the history of the faint galaxy population. As summarized for example in Table A.1 of Boué et al. (2008), this slope can strongly vary from one cluster to another, and it can also depend upon the depth of the galaxy catalog considered. While most studies give slopes in the range -0.9 to -1.5 , several find faint-end slopes as steep as -2.3 . Possible reasons for such a range of values (cosmic variance of the background counts, different mass buildup histories of clusters, systematic errors in the data analysis) are discussed in Boué et al. (2008) so are not repeated here (see references to the literature in this paper). The faint end slope can also depend on the chosen filter, but there is no clear evidence that the steepest slopes are always found in the same wavelength range.

\footnotetext{
* Based on observations obtained at the European Southern Observatory, program ESO 68.A-0084(A), P. I. E. Slezak. This research has made use of the NASA/IPAC Extragalactic Database (NED), which is operated by the Jet Propulsion Laboratory, California Institute of Technology, under contract with the National Aeronautics and Space Administration.
}

Another interesting property is that, in a given cluster, the faint end slope of the GLF has been observed to vary from one region to another. For example, this effect has been observed in several clusters such as Coma (Lobo et al. 1997; Beijersbergen et al. 2002; Adami et al. 2007a) or Abell 496 (Boué et al. 2008), and it was shown that the faint-end slope gives indications of the cluster formation history (see e.g. Adami et al. 2007a, and references therein).

We present here the analysis of the cluster Abell 780, at a redshift $z=0.0539$ (NED data base), which appears to be very relaxed and with no clear substructure (Burns et al. 1994). This cluster is better known as Hydra A, because its cD galaxy coincides with the strong wide-angle tail radio galaxy Hydra A (Owen et al. 1992). Hydra A is well known in X-rays, in particular because it is X-ray luminous (David et al. 1990 give $L_{\mathrm{X}}(1.0 \mathrm{Mpc})=4 \times 10^{44} \mathrm{erg} \mathrm{s}^{-1}$ in the $0.5-4.5 \mathrm{keV}$ band for a redshift of 0.0522 and a Hubble constant $H_{0}=70 \mathrm{~km} \mathrm{~s}^{-1} \mathrm{Mpc}^{-1}$ ) and rather hot, in spite of being a poor cluster of richness 0 (Batuski et al. 1991). The gas temperature is about $4 \mathrm{keV}$ $\left(k T_{\mathrm{X}}=4.5 \pm 0.7 \mathrm{keV}\right.$ from Einstein data, David et al. 1990, $k T_{\mathrm{X}}=3.80 \pm 0.12 \mathrm{keV}$ from ROSAT data, Mohr et al. 1999). On large scales, the thorough analysis of a deep XMM-Newton image shows that the cluster indeed appears quite smooth and relaxed (Simionescu et al. 2009a,b). On the other hand, on much smaller scales near the centre, deep Chandra data have revealed a number of interesting features in this cluster: the hot gas in its innermost regions shows cavities and Swiss cheese like topology, 
Table 1. Summary of the observations (including coordinates of the two image centres).

\begin{tabular}{lllll}
\hline \hline & East & & West \\
\hline RA (2000.0) & 139.896093 & & 139.354152 & \\
Dec (2000.0) & -12.2503 & & -12.25057 & \\
\hline & $B$ & $R$ & $B$ & 1200 \\
Exp. time (s) & 1500 & 1200 & 1500 & 1.216 \\
Airmass & 1.084 & 1.049 & 1.138 & 0.90 \\
Seeing (arcsec) & 0.90 & 1.15 & 0.95 & 2399 \\
Gain (e-/ADU) & 2999 & 2399 & 2999 & 24.334 \\
Zero Point & 24.486 & 24.334 & 24.486 & \pm 0.005 \\
ZP error & \pm 0.009 & \pm 0.005 & \pm 0.009 & \\
\hline
\end{tabular}

suggesting matter outflow. Evidence for the presence of a supermassive black hole in the central cD galaxy has also been found (McNamara et al. 2000; Wise et al. 2007).

On the other hand, very little has been published for this cluster at optical wavelengths: two galaxy redshifts (Batuski et al. 1991), and some imaging yet largely unpublished from the WINGS survey (Fasano et al. 2006). No substructures have been detected in Abell 780 from this survey (Ramella et al. 2007). However, some galaxy redshift measurements are available in NED. We present here optical images of Abell 780 in the $B$ and $R$ bands covering a field of view of $67.22 \times 32.94 \mathrm{arcmin}^{2}$, or $4.235 \times 2.075 \mathrm{Mpc}^{2}$.

The paper is organized as follows. We present our data and data reduction in Sect. 2, and describe how the final galaxy cata$\log$ is obtained. In Sect. 3, we describe the color-magnitude relation and GLFs obtained in the two bands, using the VVDS survey F02 field as a comparison field for statistical subtraction of the foreground and background galaxy populations (McCracken et al. 2003). In Sect. 4, based on galaxy redshifts found in the NED database, we analyze the environment of Abell 780 on a very large scale, and search for gravitationally bound structures by applying the Serna-Gerbal method.

To facilitate comparisons with the X-ray results by Wise et al. (2007), we assume for Abell 780 a redshift $z=0.0539$, and a flat $\Lambda \mathrm{CDM}$ cosmology with $H_{0}=70 \mathrm{~km} \mathrm{~s}^{-1} \mathrm{Mpc}^{-1}$, $\Omega_{\mathrm{M}}=0.3$, and $\Omega_{\Lambda}=0.7$, yielding a luminosity distance of $240 \mathrm{Mpc}$, a linear scale of $1.05 \mathrm{kpc} \operatorname{arcsec}^{-1}$, and a distance modulus of 36.90. In the absence of a direct estimate of the galaxy velocity dispersion $\sigma_{\mathrm{v}}$, we computed $\sigma_{\mathrm{v}}$ from the X-ray temperature measured by ROSAT applying the relation between $\sigma_{\mathrm{v}}$ and $k T$ given by Girardi et al. (1996): $\sigma=10^{2.59 \pm 0.04} T^{0.50 \pm 0.07}$. For $k T=3.80 \mathrm{keV}$, we obtain $\sigma_{\mathrm{v}}=758 \mathrm{~km} \mathrm{~s}^{-1}$. We then calculate the $r_{200}$ radius using Eq. (8) from Carlberg et al. (1997) and find $r_{200}=1.91 \mathrm{Mpc}$.

\section{Data and methods}

\subsection{The data}

We observed the cluster Abell 780 with the ESO 2.2m telescope and the WFI camera on March 18, 2002 (ESO Program 68.A-0084(A)). Two fields were observed, covering the east and west parts of the cluster, in both Johnson $B$ and $R$ filters (ESO filters $B-842$ and $R-844$ ). The observations are summarized in Table 1 . The camera comprises $8 \mathrm{CCDs}$, giving a field of about $8750 \times 8305$ pixels with a pixel size of $0.238 \times 0.238 \mathrm{arcsec}^{2}$. Each WFI field is therefore $34.708 \times 32.943 \mathrm{arcmin}^{2}$, or $2187 \times$ $2075 \mathrm{kpc}^{2}$ at the cluster distance.

The two fields have a small area in common 2.19 arcmin wide in right ascension (131.5 arcsec, or 552.5 pixels), where

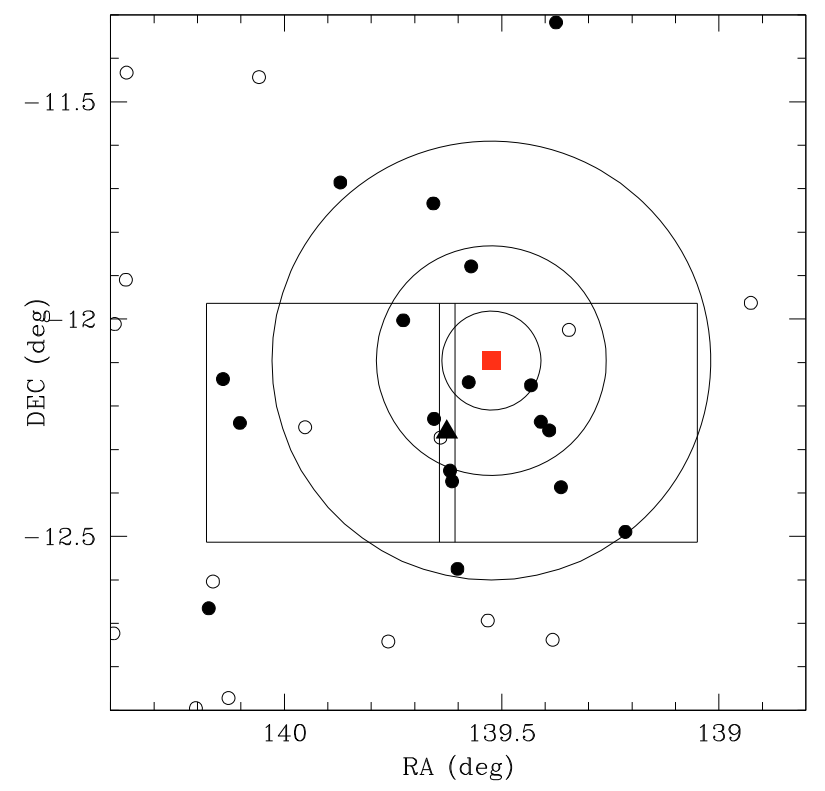

Fig. 1. Map of the region of Abell 780. The two squares show the positions of our images. The black triangle shows the Abell 780 cluster position according to the NED and Simbad databases, and the red square that of the cluster centre as we define it (coinciding with the Hydra A $\mathrm{cD}$ galaxy, see text). The three concentric circles show radii of $0.5,1.0$, and $1.91 \mathrm{Mpc}\left(r_{200}\right)$. Small circles show galaxies with redshifts taken from NED, with filled circles indicating galaxies in the [0.0338, 0.0738] redshift interval corresponding to a broad velocity interval around the mean cluster velocity.

we believed that the cluster centre was at the time of the observations (these were the coordinates given for Abell 780 in the NED and Simbad databases). However, we found out later that the position of the $\mathrm{cD}$ galaxy (the Hydra A galaxy), which coincides with the maximum X-ray emission, is offcentered by 6.1 arcmin in right ascension and 9.9 arcmin in declination towards the northwest. We will take the position of the $\mathrm{cD}$ as the cluster centre hereafter: $\alpha(2000.0)=139.52375 \mathrm{deg}$, $\delta(2000.0)=-12.0955 \mathrm{deg}$. Our mapping of the cluster therefore misses part of its North half, but on the other hand allows us to probe the South half of the cluster further away from the centre (see Fig. 1). A larger fraction of the cluster is included in the west field than in the east one. The total area covered by our two fields is $67.22 \times 32.94 \mathrm{arcmin}^{2}$, or $4.235 \times 2.075 \mathrm{Mpc}^{2}$ in right ascension $\times$ declination.

\subsection{Data reduction and source extraction}

The images were bias- and flatfield-corrected in the usual way, using flat fields obtained at twilight and dawn on the sky. For each field, 5 individual exposures were obtained with some dithering. They were combined after being individually corrected for bias and flat field. We thus obtained a final set of four images in two bands, plus their corresponding weight maps. These weight maps allow us to take into account the fact that all the pixels in each image are not similarly exposed because of the dithering applied during such observations. The SExtractor software takes them into account when detecting and measuring objects on the images. Seeing measurements were made on unsaturated stars on the four final stacked images. Since the east and west images had slightly different seeings (at least in the $R$ band), they were processed independently (see Table 1). 
Photometric zero points were estimated from the observation of standard stars from the Landolt list. Masks were made to exclude the regions with saturated pixels and bright stars and at the CCD edges. Object detection was performed by applying the SExtractor software (Bertin \& Arnouts 1996). Detections were made in the $R$ band (which is more sensitive), and then measurements were made in the $B$ band with the double image mode. After trying several values, we adopted a detection threshold of $1.5 \sigma$ and a minimum number of 5 pixels required for an object to be detected.

Total magnitudes (MAG-AUTO given by SExtractor) were computed in the Vega system. Star catalogues were left in the Vega system in order to be directly comparable with the Besançon counts (see Sect. 2.5). Galaxy magnitudes were converted to the $A B$ system so that galaxy counts were directly comparable to those of the VVDS (see below). For this we applied the following formulae ${ }^{1}: B(A B)=B$ (Vega) -0.163 and $R(A B)=R($ Vega $)+0.055$.

All magnitudes were corrected for galactic extinction, which is not negligible since Abell 780 is at relatively low galactic latitude $\left(b=25^{\circ}\right)$. The Schlegel et al. (1998) extinction maps in the direction of our two fields show the same mean extinction (within the dispersion), with flux variations of only $11 \%$ within each image, corresponding to magnitude variations of at most 0.05 in $B$ and 0.03 in $R$, adopting a mean extinction value. We therefore adopted constant extinction factors for both fields: 0.214 mag in $B$ and 0.132 mag in $R$. In view of the small redshift of Abell 780, we did not apply any $K$-correction. We thus obtained catalogues of objects in $B$ and $R$ for the east and west fields separately.

\subsection{Catalogue completeness}

The completeness of the catalogue was estimated by simulations. For this, we added artificial stars of different magnitudes to the CCD images and then attempted to recover them by running SExtractor again with the same parameters as were used for object detection and classification on the original images. In this way, the completeness is measured on the original images and at different locations in the cluster.

In practice, we divided the full field of view into eight subimages, each $4030 \times 4030$ pixels $^{2}$ (similar to the subimage sizes already adopted e.g. in Adami et al. 2006a). This represents a good compromise between the spatial resolution of the completeness maps that we compute and the Poissonian error bars directly driven by the numbers of objects considered in each subregion. Choosing eight subareas also allowed us to detect potential completeness variations from one WFI CCD to another.

In each subfield and for each $0.5 \mathrm{mag}$ bin between $R=20$ and 26, we generated and added to the image one star that we then tried to detect with SExtractor, assuming the same parameters as previously. This process was repeated 100 times for each of the eight subfields.

The seeing variations between the east and west images and in the $R$ and $B$ bands are taken into account. We also corrected magnitudes for extinction as for our object catalogue, and transformed them into $A B$ magnitudes. Such simulations give a completeness percentage for stars. This is obviously an upper limit for the completeness level, since stars are easier to detect than galaxies. However, we showed in a previous paper that this method gives a good estimate of the completeness for normal

\footnotetext{
1 http://WwW . astro. utoronto.ca/ patton/astro/mags. html\#conversions
}
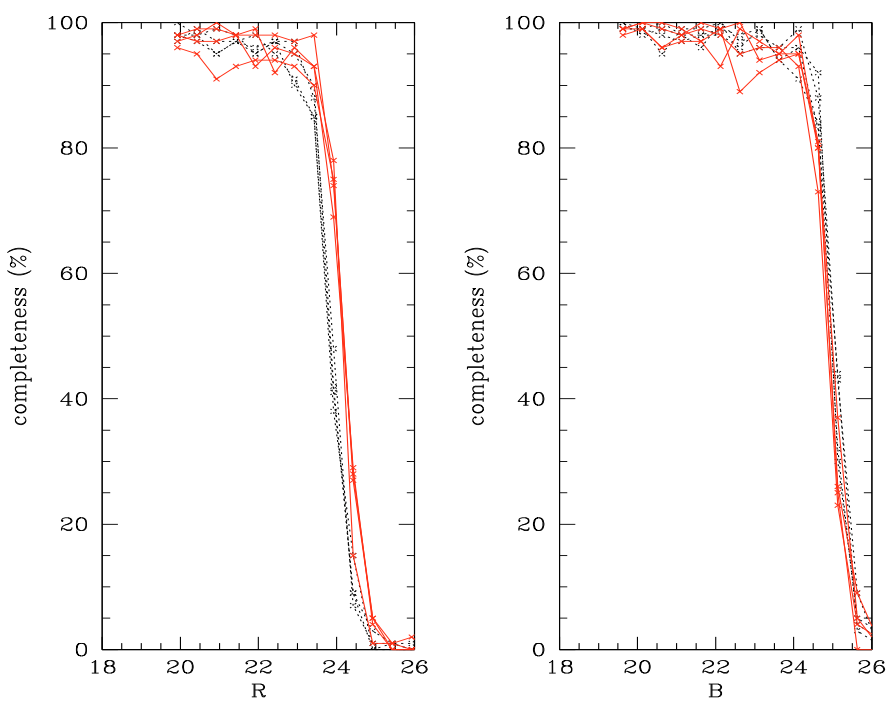

Fig. 2. Completeness in percentages in $B$ (right) and $R$ (left) for pointlike objects. The black dotted lines correspond to the east half of the total field and the red full lines to the west half (where the cluster is mainly located).

galaxies if we apply a shift of $\sim 0.5 \mathrm{mag}$ (see Adami et al. 2006a). This does not apply to low surface brightness galaxies, which are not expected to be detected here, mainly because they would be fainter than our detection limit, as derived from our results for LSBs in Coma (see Adami et al. 2006a,b). Results are shown in Fig. 2.

From these simulations, and taking into account that results are worse by $\sim 0.5 \mathrm{mag}$ for mean galaxy populations than for stars, we can consider that our galaxy catalogue is complete to better than $90 \%$ for $R \leq 23$ and $50 \%$ for $R \leq 23.5$. In the $B$ band, the corresponding numbers for $90 \%$ and $50 \%$ completeness are $B \leq 23.7$ and $B \leq 24.3$, respectively.

\subsection{Star-galaxy separation}

To separate stars from galaxies, we plotted the central surface brightness in the $R$ band as a function of $R$ magnitude for the east and west fields (Fig. 3). Stars show well-defined sequences bound by lines with slopes of 1.00 .

To estimate the ordinate positions of the two upper lines (separating stars from galaxies), we considered the star simulations described in Sect. 2.3. for the $R$ band. With respective seeings in the $R$ band of 1.15 and 0.90 arcsec in the east and west regions, the $1 \sigma$ dispersions of the star distributions around a mean line of slope 1.00 are 0.127 and 0.091 , respectively. We consider that stars are located at $\pm 3 \sigma$ around the best line fit. The corresponding ordinate positions for $R=15$ of the two upper lines (those separating stars from galaxies) are 15.61 and 15.22 for the east and west plots, respectively. All the objects above these lines have been classified as galaxies.

We looked individually at some objects located at the top edge of the quoted sequence in the $R=18-20$ mag interval to check that this separation was correct. A few objects are visible in Fig. 3 at $\left(R<22, \mu_{R}>24\right)$. They do not correspond to low surface brightness galaxies but are all tiny defects in the CCDs.

A number of "objects" appear to form a second, broader sequence below the stars. On the images, these objects are found to be false detections and are in a great majority located at the CCD edges or at the edges of the masks drawn to cover bright stars. As seen in Fig. 3, these defects can be separated from stars by the 

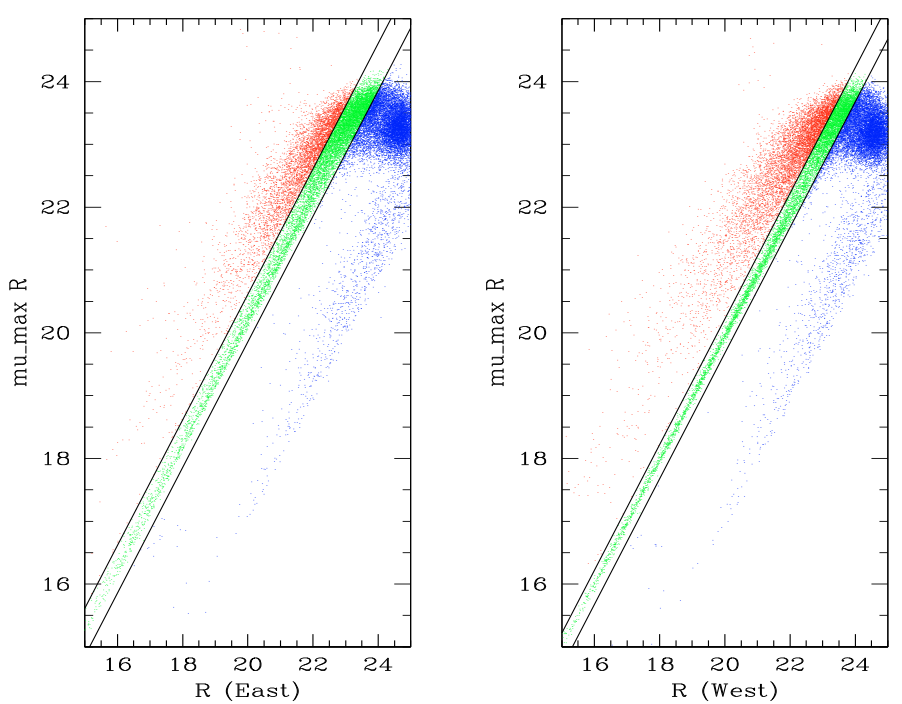

Fig. 3. Central surface brightness in the $R$ band as a function of $R$ magnitude for the East (left) and West (right) fields. The lines separate the galaxies (red points, above the top lines) from the stars (green points, between the two lines). Objects below the bottom lines (blue points) have been considered as defects. Objects with $R<22$ and $\mu_{R}>24$ are defects as well.

two lower lines delimiting the star region, taken to be symmetric of the star-galaxy separation line defined above relatively to the line that fits the star sequence best. The objects below these two lower lines have been considered as defects and are eliminated from our catalogues hereafter.

This classification into stars, galaxies, and defects is of course not valid over our whole range of magnitudes because of seeing limitations; for example, galaxy and star sequences appear to merge above $R \sim 21.5$ in Fig. 3 . We also checked by eye on the images that most of the 260 objects brighter than $R=18$ and classified as galaxies were indeed galaxies and not saturated stars.

\subsection{Star counts}

To estimate more precisely the reliability of our star-galaxy separation, we retrieved the star catalogue from the Besançon model for our Galaxy (Robin et al. 2003) in a $1 \mathrm{deg}^{2}$ region centered on the position of Abell 780 given by NED (139.6265 deg, $-12.2611 \mathrm{deg}, \mathrm{J} 2000.0$ ). As seen from Fig. 4, the star count histograms in the east and west regions estimated in Sect. 2.4 agree with the Besançon model star counts, and do so very well in the west region and within Poissonian error bars for the east region, between magnitudes $R \sim 17$ and $R \sim 21.5$. At brighter magnitudes, the histograms do not coincide, both because we masked very bright stars and because our total field is relatively small, leading to small number statistics for bright stars.

At faint magnitudes $(R \geq 21.5)$, our star counts rise steeply, while the Besançon model counts increase much more smoothly. As expected, this implies that our star-galaxy separation is not reliable at magnitudes over $R=21.5$ (or, to state this differently, the 21.0-21.5 bin is the faintest magnitude bin where the stargalaxy separation can be considered as reliable).

\subsection{Galaxy counts}

To perform galaxy counts, we build a single catalogue of the full east+west field after eliminating objects in common. We kept the
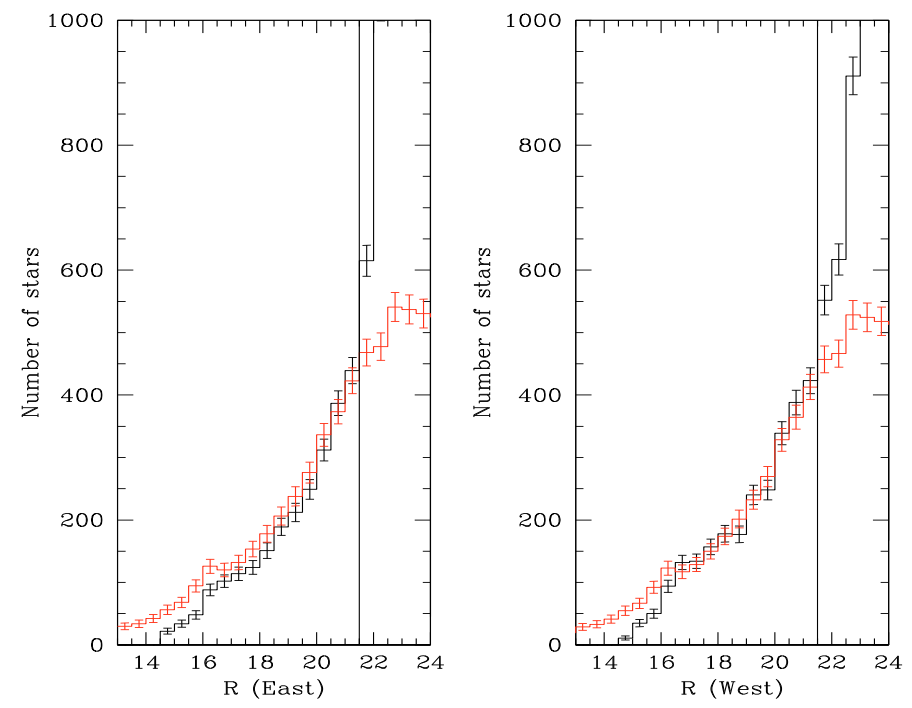

Fig. 4. Black histograms: star counts in the direction of Abell 780 in the east (left) and west (right) fields. The red histograms show the star counts from the Besançon models normalized to the respective surfaces of our fields. Error bars are Poissonian. The vertical lines at $R=21.5$ show the limit beyond which our star-galaxy separation is not reliable.

Table 2. Percentage of stars in bins of $0.5 \mathrm{mag}$ in the east and west fields, and average percentage of galaxies.

\begin{tabular}{llll}
\hline \hline$R$ & $\begin{array}{l}\text { Stars } \\
\text { east }\end{array}$ & $\begin{array}{l}\text { Stars } \\
\text { west }\end{array}$ & $\begin{array}{l}\text { Galaxies } \\
\text { average }\end{array}$ \\
\hline 21.75 & $30.3 \%$ & $29.6 \%$ & $70.0 \%$ \\
22.25 & $22.4 \%$ & $23.9 \%$ & $76.8 \%$ \\
22.75 & $20.9 \%$ & $21.8 \%$ & $78.6 \%$ \\
23.25 & $20.8 \%$ & $19.5 \%$ & $79.8 \%$ \\
\hline
\end{tabular}

objects measured in the West field, since this is the field containing a higher fraction of the cluster, and also the field with the best seeing. We consider the star-galaxy separation described above to be reliable for $R<21.5$. Our full galaxy catalogue (with the east and west regions merged) will be publicly available at the following address: http://cencosw. oamp. fr/. It comprises 4256 galaxies brighter than $R=21.5$.

For fainter magnitudes, we estimated the percentage of stars by comparing the total number of detected objects (stars+galaxies) to the predictions of the Besançon model in bins of 0.5 mag. We give these percentages in Table 2. The average fraction of galaxies was estimated in each magnitude bin from the average of the star percentages.

For $R \geq 21.5$ we therefore consider that in each magnitude bin the number of galaxies is equal to the total number of objects (stars + galaxies) multiplied by the average percentage of galaxies. We do not consider galaxy counts above $R=23.5$, since incompleteness becomes too strong.

Galaxy counts were made in four regions defined by their distance $r$ to the cluster centre in kpc: a circle of radius $r<$ $500 \mathrm{kpc}$, a circle of radius $r<1000 \mathrm{kpc}$, a ring between 500 and $1000 \mathrm{kpc}$, and a ring between 1000 and $1500 \mathrm{kpc}$. Except for the first, all of these regions are truncated since our images do not fully cover them (see Fig. 1). The areas covered by each of these four regions after masking bright stars are given in Table 3. Galaxy counts are displayed in Fig. 5. Error bars are Poissonian. 
Table 3. Area effectively covered by each of the four regions of Fig. 5 .

\begin{tabular}{ll}
\hline \hline Region & $\begin{array}{l}\text { Area } \\
\left(\mathrm{deg}^{2}\right)\end{array}$ \\
\hline$r<500 \mathrm{kpc}$ & 0.049321 \\
$r<1000 \mathrm{kpc}$ & 0.17635 \\
$500<r<1000 \mathrm{kpc}$ & 0.12261 \\
$1000<r<1500 \mathrm{kpc}$ & 0.167136 \\
\hline
\end{tabular}

\subsection{Comparison field}

To perform a statistical subtraction of the background contribution, we consider the galaxy counts in the F02 VVDS field ${ }^{2}$, which was observed with the same filters as Abell 780 and is prone to little extinction since it is located at high galactic latitude $\left(58.0^{\circ}\right)$. It covers a total area of $1.18 \mathrm{deg}^{2}$ and is fully complete and free of surface brightness selection effects up to $I_{\mathrm{AB}}=24$, with more than $50 \%$ completeness at $B=26.5$ and $R=25.9$ (see McCracken et al. 2003, Sect. 4.1).

We take the galaxy counts in 0.5 mag intervals normalized to $1 \mathrm{deg}^{2}$ directly from Table 2 of McCracken et al. (2003). Since our magnitudes were transformed to the AB system, the comparison of the galaxy counts is straightforward. The VVDS galaxy counts are shown in Fig. 5, where all counts are normalized to $1 \mathrm{deg}^{2}$. We can see from this figure that it is only in the $r<500 \mathrm{kpc}$ region that the cluster galaxy counts are notably above the background counts up to $R \sim 23.5$. In the $500<r<1000 \mathrm{kpc}$ and $1000<r<1500 \mathrm{kpc}$ regions, there is overall agreement between the galaxy counts and those of the VVDS, implying that the cluster does not show strongly above the background for $r>500 \mathrm{kpc}$. This explains why the galaxy counts in a $r<1000 \mathrm{kpc}$ circle are so strongly diluted that they are not significantly higher than the background counts.

We therefore only analyze the galaxy luminosity function in the $r<500 \mathrm{kpc}$ region hereafter.

\section{Results: colour-magnitude diagram and galaxy luminosity functions}

\subsection{Colour-magnitude diagram}

Since the McCracken et al. (2003) galaxy counts that we use to subtract the background contribution start at $R=18$, we need a selection criterium to build the GLFs for $R<18$.

The $B-R$ vs. $R$ colour-magnitude diagram is shown in Fig. 6 for the same four regions as in Fig. 5. A sequence is well-defined for galaxies in the magnitude range $15<R<18.5$, particularly in the plot corresponding to a radial distance to the cluster centre smaller than $500 \mathrm{kpc}$. We computed the best fit to the $B-R$ vs. $R$ relation in this zone and in this magnitude range by applying a simple linear regression. We then eliminated the galaxies located at more than $3 \sigma$ from this relation and computed the $B-R$ vs. $R$ relation again. The equation of the colour-magnitude relation is found to be: $B-R=-0.064 R+2.27$ with a $\pm 2 \sigma$ scatter of \pm 0.28 . We consider that all the galaxies located within $2 \sigma$ of this relation (i.e. between the two red lines of Fig. 6) belong to the cluster. An eye check of the 5 very bright objects $(R<15)$ below the sequence shows that 3 are actually stars with small diffraction crosses (misclassified by SExtractor as galaxies), one is the blend of a bright galaxy plus a star, and one is a star with one or two objects superimposed.

\footnotetext{
${ }^{2}$ http://cencosw. oamp. fr/VVDSphot/FQ2
}
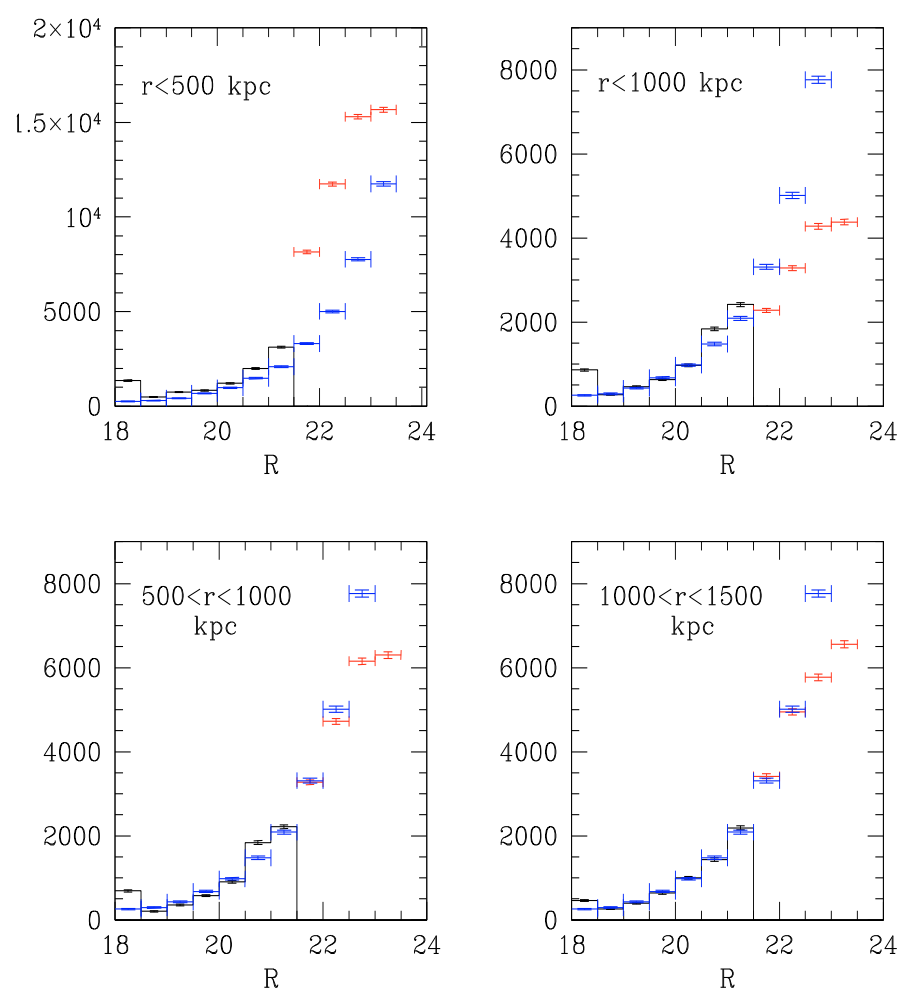

Fig. 5. Galaxy counts in four regions defined by their distance $r$ to the cluster center in kpc. Top left: circle of radius $r<500 \mathrm{kpc}$; top right: circle of radius $r<1000 \mathrm{kpc}$; bottom left: ring between 500 and $1000 \mathrm{kpc}$; bottom right: ring between 1000 and $1500 \mathrm{kpc}$. The black histograms correspond to galaxies selected as in Fig. 3 and red histograms correspond to galaxy counts estimated statistically. The blue histograms show the VVDS counts as described in Sect. 2.7. Error bars are Poissonian. All counts are normalized to $1 \mathrm{deg}^{2}$.
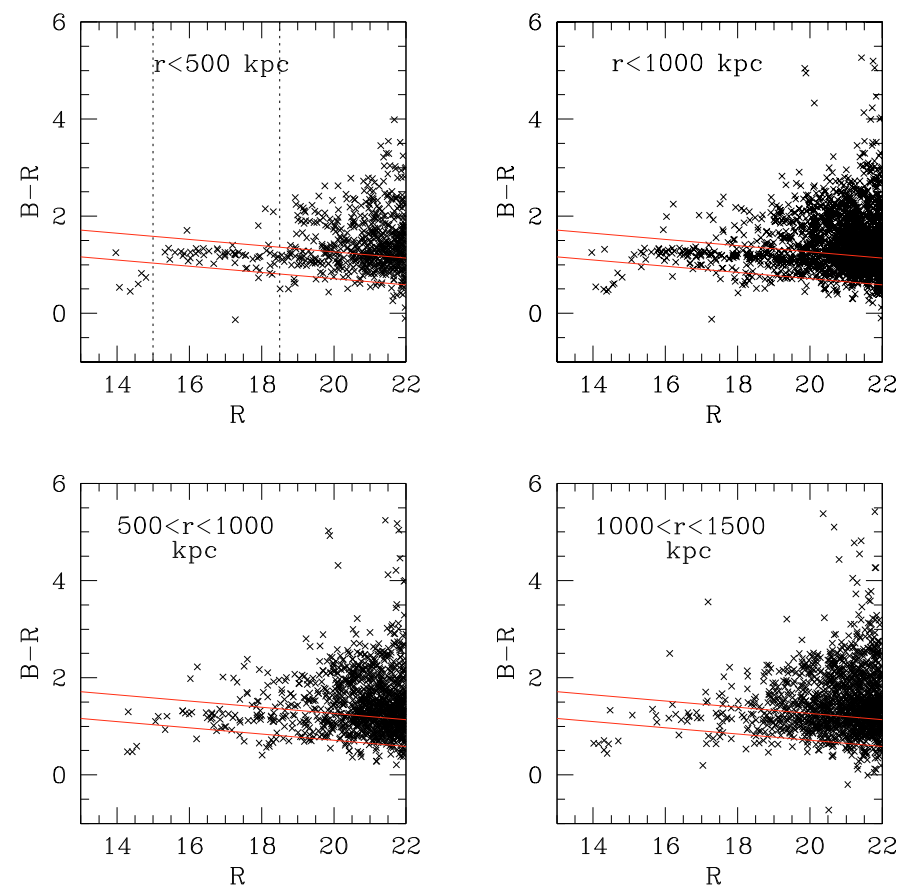

Fig. 6. $B-R$ vs. $R$ colour-magnitude diagram in the same regions as Fig. 5. The red lines show the interval in which galaxies are considered as belonging to Abell 780. The vertical dotted lines on the top left figure indicate the magnitude interval in which the colour-magnitude relation was computed. 
Table 4. Galaxy counts (in 0.5 mag bins) and luminosity functions in the $B$ and $R$ bands in a region within $500 \mathrm{kpc}$ of the cluster centre, normalized to a $1 \mathrm{deg}^{2}$ area (numbers are not given when the GLF is negative).

\begin{tabular}{rrrrrr}
\hline \hline$B$ & $N B_{\text {nor }}$ & $G L F_{B}$ & $R$ & $N R_{\text {nor }}$ & $\mathrm{GLF}_{R}$ \\
\hline 13.75 & & 0 & 13.75 & & 20 \\
14.25 & & 0 & 14.25 & & 0 \\
14.75 & & 0 & 14.75 & & 0 \\
15.25 & & 20 & 15.25 & & 61 \\
15.75 & & 0 & 15.75 & & 101 \\
16.25 & & 0 & 16.25 & & 162 \\
16.75 & & 81 & 16.75 & & 162 \\
17.25 & & 162 & 17.25 & & 203 \\
17.75 & & 122 & 17.75 & & 223 \\
18.25 & & 264 & 18.25 & & 203 \\
18.75 & 223 & 157 & 18.75 & 487 & 192 \\
19.25 & 223 & 123 & 19.25 & 750 & 323 \\
19.75 & 304 & 156 & 19.75 & 831 & 155 \\
20.25 & 527 & 252 & 20.25 & 1217 & 240 \\
20.75 & 487 & 60 & 20.75 & 1987 & 508 \\
21.25 & 1217 & 476 & 21.25 & 3122 & 1033 \\
21.75 & & & 21.75 & 8148 & 4837 \\
22.25 & & & 22.25 & 11743 & 6731 \\
22.75 & & & 22.25 & 15296 & 7534 \\
\hline
\end{tabular}
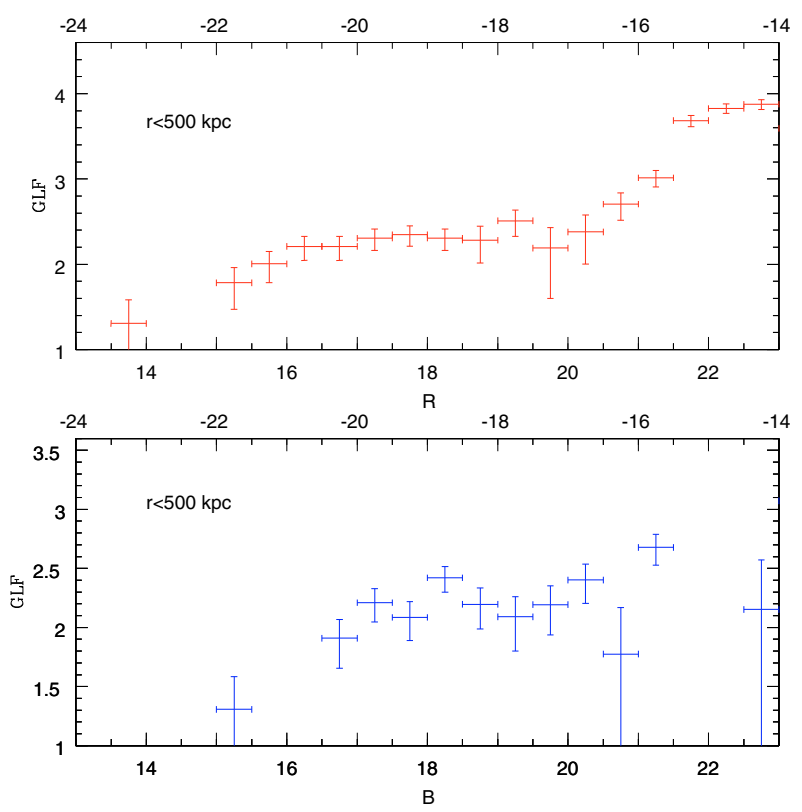

Fig. 7. Galaxy luminosity functions in a $500 \mathrm{kpc}$ radius region around the cluster center in the $R$ (top) and $B$ (bottom) bands, normalized to $1 \mathrm{deg}^{2}$, in logarithmic scale. Apparent and absolute magnitudes are indicated in the bottom and top scales, respectively.

\subsection{Global galaxy luminosity functions}

Galaxies belonging to Abell 780 with magnitudes $R \leq 18.5$ were selected by applying the color-magnitude selection described above. This limit of 18.5 was chosen rather than the lower magnitude limit of 18.0 of the VVDS counts to avoid possible errors due to small number statistics in the very first VVDS bin. Numbers of galaxies were computed in bins of $0.5 \mathrm{mag}$.

For galaxies with magnitudes $18.5<R<23$, GLFs were estimated in bins of 0.5 mag by simply subtracting the VVDS F02 counts from the Abell 780 counts in the $B$ and $R$ bands, estimated as described in Sect. 2.6. All counts were normalized to a $1 \mathrm{deg}^{2}$ region. Results are given in Table 4 and Fig. 7 for the region of
$500 \mathrm{kpc}$ radius around the cluster center. For magnitudes above $B=21.5$ (except for a point at $B=22.75$, which has a very large error bar, as is also the case for the point at $B=20.75$ ) and $R>23$, our galaxy counts become smaller than those in the background. The error bars drawn in Fig. 7 were taken to be 4 times the Poissonian errors on galaxy counts, as suggested by detailed simulations previously performed by our team (see Boué et al. 2008, Fig. 5).

We can see, particularly in $R$ where the signal to noise ratio is better and the GLF smoother, that the GLF is composite, with a broadly shaped distribution at bright magnitudes, and a rising part for $R>19.5$. A power law fit between $R=20.25$ and $R=$ 21.75 (corresponding to absolute magnitudes between -16.65 and -15.15) performed with a linear regression gives a slope $\alpha=-0.85 \pm 0.12$ (when expressed as a function of absolute $R$ mag).

The bright part of the GLF $\left(R<18\right.$, or $\left.M_{R}<-18.9\right)$ is very similar in shape to the GLFs recently obtained e.g. by Andreon et al. (2008), who analyzed the GLFs of a sample of clusters at various redshifts, limited to bright absolute magnitudes $M_{V}<$ -19 .

The faint end slope is somewhat flatter than usually found in clusters, where it is usually between -1.0 and -2.0 in the same absolute magnitude range (see e.g. Boué et al. 2008, Table A.1). Besides, it is somewhat surprising that the GLF flattens for $R>22$ since the completeness was estimated to be better than $90 \%$ up to $R=23$. However, the region that we consider is rather small (500 kpc in radius, corresponding to $\left.0.26 r_{200}\right)$ and corresponds to the cluster centre, where the slope is expected to be flatter. This would agree with the general picture of a relaxed cluster where the central $\mathrm{cD}$ galaxy has had time to accrete many surrounding dwarf galaxies without being refueled by numerous infalls from the field, thus leading to a smaller number of faint galaxies than expected. However, Fig. 5 seems to show that, if there are any dwarfs left, then they are in the center, contradicting the previous explanation. One possible reason (qualitatively supported for example by Adami et al. 2007b) could be that faint galaxies in clusters (i.e. here $R>20$ or $M_{R}>-17$ ) could have different origins. Moderately faint galaxies $(20<R<22)$ could be classically accreted faint field galaxies, while fainter objects $(R>22)$ could be cluster-made galaxies or, in other words, the debris of larger galaxies. In this picture, we can explain the rising GLF of Abell 780 up to $R \sim 22$ as being driven by normal accreted field galaxies. The flatter GLF at $R>22$ would then be explained by the quite isolated and quiet nature of A780, likely to reduce interaction processes and therefore to inhibit the creation of faint debris inside the cluster. However, this scenario, proposed for the Coma cluster, may not be valid here, since it does not explain why there are fewer moderately faint galaxies in the region between 500 and $1000 \mathrm{kpc}$ than in the innermost $500 \mathrm{kpc}$ radius region.

A comparison of the $R$ band GLF of Abell 780 (in the region of $500 \mathrm{kpc}$ radius) with the GLFs in the north and south halves of the Coma cluster (Adami et al. 2007a) shows that the GLF shapes are quite similar up to the absolute magnitude $M_{R} \sim-14.5$. However, our data are not as deep as those of Coma, so we cannot compare the very faint ends, corresponding to $M_{R}>-14.5$ (where the Coma north and south GLFs differ, due to the influence of the environment on a large scale).

The GLF in the $B$ band does not show any obvious difference with that in the $R$ band; since it is much noisier, we will not discuss it further. 

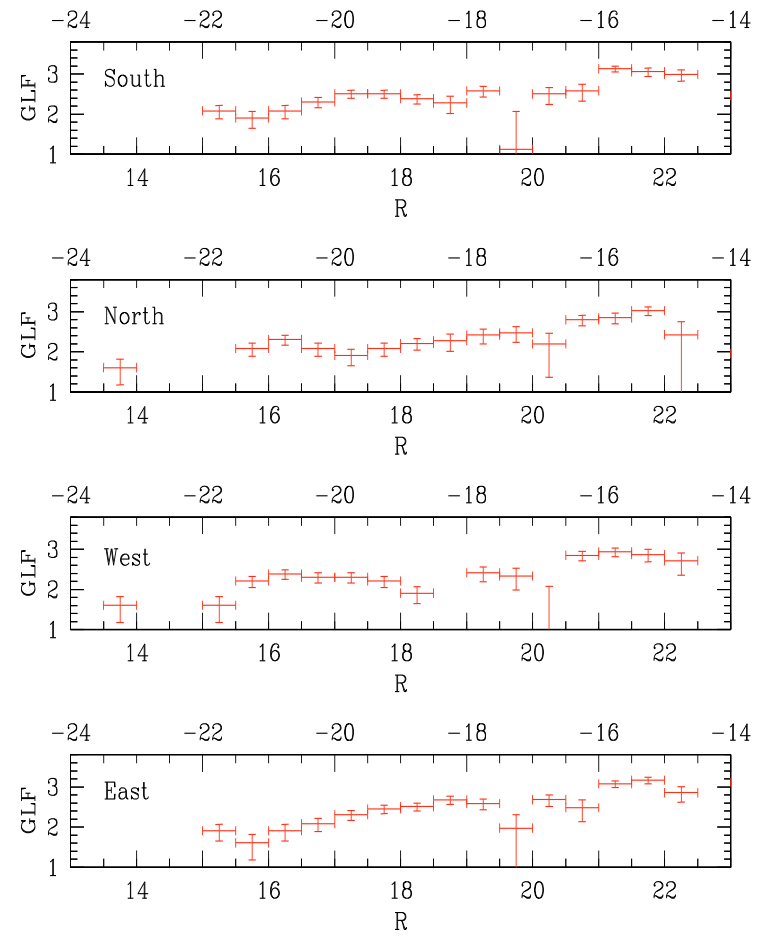

Fig. 8. Galaxy luminosity functions in the $R$ band normalized to an area of $1 \mathrm{deg}^{2}$. The $500 \mathrm{kpc}$ radius region around the cluster centre was divided in halves: from top to bottom south half, north half, west half and east half.

\subsection{Galaxy luminosity functions in various regions}

In order to see if any difference suggesting environmental effects could be found, we analyzed the GLF in various regions of the cluster. For this, we cut the $500 \mathrm{kpc}$ radius region into two halves: one north and one south of the cluster center, then two other halves: one east and one west of the cluster centre. The GLFs were derived as described above in those four regions, in the $R$ band only since it is the band with the highest number of galaxy counts. The GLFs obtained are given in Fig. 8 (normalized to an area of $1 \mathrm{deg}^{2}$ ). We can see that in the west and south zones their shapes are more similar to the overall cluster GLF than in the east and north where the GLFs rise more or less continuously, but it is difficult to say much more since they are rather noisy.

Altogether, environmental effects appear rather weak in Abell 780. We describe below a short analysis of the Abell 780 cluster environment, performed to look for apparent substructures such as filaments along which galaxy infall could be occurring.

\section{Discussion and conclusions}

\subsection{The central regions of the cluster: comparison with $X$-ray data}

Abell 780 is well known in X-rays under the name of Hydra A, originating from the cluster $\mathrm{cD}$ being a strong radio emitting galaxy bearing this name. Based on a deep Chandra image, Wise et al. (2007) found several X-ray cavities (labeled A to F) in the central region, which they explained by outflow (continuous or in bursts) from the nucleus of the central galaxy. The cavities could each harbor one or several AGN (expected to be

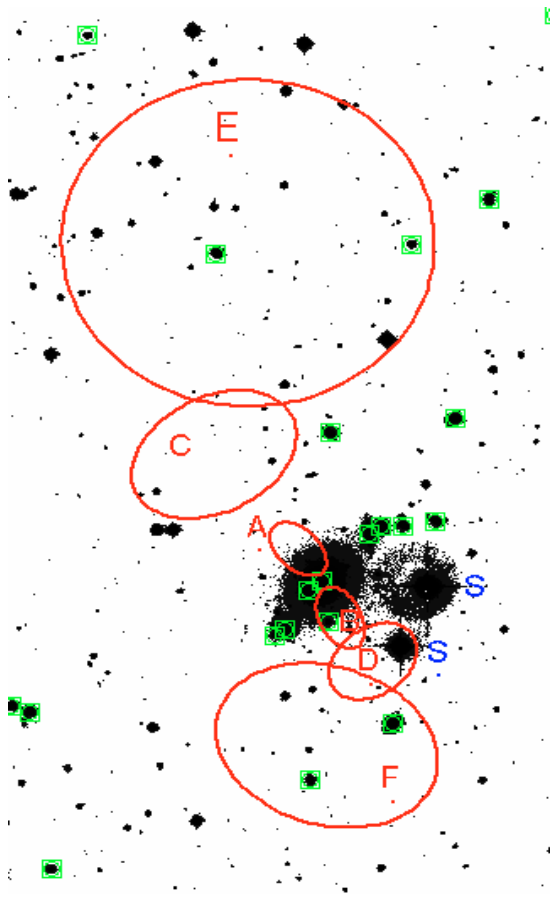

Fig. 9. Inverted colour $R$ band image of the cluster centre with the positions of the X-ray cavities found by Wise et al. (2007) superimposed and labeled in red. The $\mathrm{S}$ symbol shows the positions of the two bright stars close to the $\mathrm{cD}$. Galaxies brighter than $R=18$ are indicated with a green square. North is top and east is left.

intrinsically bright and to have bluer colours than normal galaxies). The optical $R$ band image around the cluster centre is shown in Fig. 9 with the positions of the X-ray cavities from Wise et al. superimposed. Cavities A and B are partly superimposed on the $\mathrm{cD}$, and SExtractor detects several galaxies brighter than $R=18$ superimposed on the $\mathrm{cD}$ (but slightly outside $\mathrm{A}$ and $\mathrm{B}$ ). No bright galaxy is visible in cavities $\mathrm{C}$ and $\mathrm{D}$. Cavity E contains two objects brighter than $R=18$, but one of them is probably a star (a weak diffraction cross is visible when carefully looking at the image). Cavity $\mathrm{F}$ has two bright galaxies with $R$ mag 16.2 and 16.8; the brighter one has a redshift $z=0.051526$ according to NED, but all three galaxies in cavities $\mathrm{E}$ and $\mathrm{F}$ have normal $B-R$ colours, so either spectroscopy or high-resolution multiband imaging (to search for blue cores) are necessary to check that they are AGN. To our knowledge they have no X-ray or radio counterparts.

\subsection{Large-scale structure}

Since clusters are believed to form from material falling along filaments, environmental effects are expected to have a strong influence on their properties. We have searched the NED database in the environment of Abell 780 and found 1219 galaxies with measured redshifts in a region of $5 \mathrm{deg}(18.9 \mathrm{Mpc})$ radius around the cluster. Out of these, 719 have redshifts in the $[0.0338,0.0738]$ range, i.e. within $\pm 6000 \mathrm{~km} \mathrm{~s}^{-1}$ of the mean velocity of Abell 780, a broad velocity interval corresponding to $\pm 8 \sigma_{\mathrm{v}}$, chosen to avoid "losing" galaxies that could be linked to Abell 780. To search for gravitationally bound structures, we selected a subsample of galaxies that also had magnitudes available, and the spatial distribution of these galaxies is shown in Fig. 10. The Abell 754 cluster is clearly visible northwest of Abell 780, at a distance of about $12.8 \mathrm{Mpc}$, and its redshift 

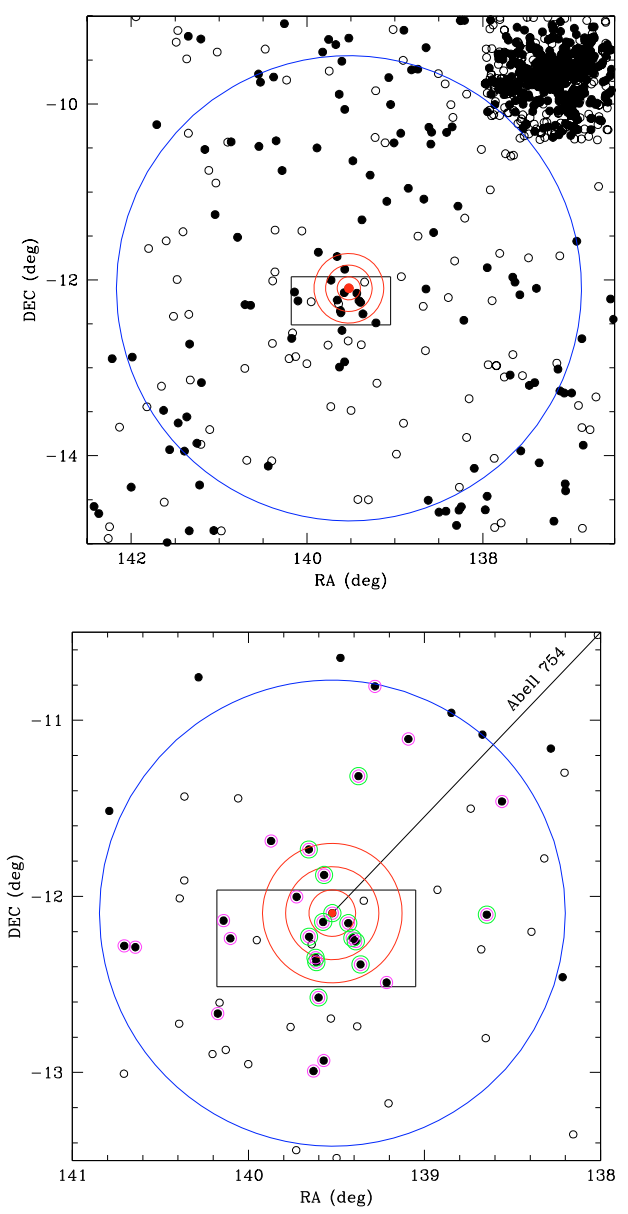

Fig. 10. Positions of the galaxies with measured redshifts and magnitudes in a large-scale region around Abell 780, taken from NED. Small filled black circles show galaxies with redshifts in the [0.0338, 0.0738] redshift interval, while empty circles show galaxies with redshifts outside this range. The black rectangle indicates the total field covered by our images. The red filled square corresponds to the cluster centre as defined in the text. The three small red concentric circles correspond to radii of $0.5,1.0$, and $1.5 \mathrm{Mpc}$. Top figure: the large blue circle corresponds to a radius of $10.0 \mathrm{Mpc}$. The Abell 754 cluster is visible at the top right. Bottom figure: zoom of the top figure, with the large blue circle having a radius $5.0 \mathrm{Mpc}$. The 27 galaxies circled in magenta and the 14 galaxies circled in green correspond to the two gravitationally bound structures according to the Serna-Gerbal analysis. The line indicates the direction towards Abell 754.

is 0.0542 , almost the same as that of Abell 780. However, no galaxy filament is detected between Abell 780 and Abell 754 .

In two smaller regions of radii $10 \mathrm{Mpc}$ and $5 \mathrm{Mpc}$ around Abell 780, there are 202 and 85 galaxies with measured redshifts (not all with measured magnitudes), with 107 and 58 galaxies in the $[0.0338,0.0738]$ redshift range, respectively. If we consider galaxies in this redshift range and cut the $5 \mathrm{Mpc}$ circle in halves, we find that there are 41, 17, 29, and 29 galaxies in the south, north, west, and east halves respectively. If we divide the cluster in halves, with the dividing line oriented perpendicular to the line connecting Abell 780 to Abell 754, we find fewer galaxies in the half circle closer to Abell 754 than in the other one (23 against 35 ). These numbers are too small for statistics, and we have no information on the NED data coverage and completeness, so all we can say is that, according to these data, there is no evidence of a galaxy density enhancement towards
Abell 754; on the contrary, there may be more galaxies south of the cluster on a large scale.

\subsection{Search for gravitationally bound structures}

To search for gravitationally bound structures, we applied the Serna-Gerbal (1996, hereafter SG) method to the redshift and magnitude catalogue that we compiled within a radius of $5 \mathrm{Mpc}$ (see above). The SG method allows gravitationally bound galaxy subgroups to be extracted from a catalogue containing positions, magnitudes, and redshifts, based on the calculation of their relative (negative) binding energies. This calculation takes into account the mass to luminosity $M / L$ ratio chosen by the user as an input value, but the group mass derived later is estimated from the group binding energy and velocity dispersion, and does not depend upon $M / L$, which only acts as a contrast criterium. We assume here that $M / L=400$. Qualitatively, high input values of $M / L$ only allow the detection of the major structures, while low values of $M / L$ allow detecting structures with low link energy. Since we only have a limited redshift and magnitude catalogue, we do not search for small structures in detail. In any case, results do not depend strongly on the value of $M / L$, since a variation of a factor of two in this parameter does not change the results significantly. However, our redshift catalogue is extracted from NED, and since we do not have completeness information, we cannot estimate quantitatively how strong the results of the SG analysis are.

The SG method gives a list of galaxies belonging to each group as output, as well as information on the binding energy and mass of the group itself, with a minimum number of 3 galaxies per group.

Within a $5 \mathrm{Mpc}$ radius, once galaxies with no magnitudes available are excluded, we are left with a catalogue of 49 galaxies with magnitudes brighter than 16 . Out of these 49 galaxies, 16 have no filter indication in NED, 21 have magnitudes in the $R$ filter, and 12 in the $I$ filter. In order to make magnitudes as homogeneous as possible, we converted the $I$ magnitudes into $R$ magnitudes assuming $R-I=0.7$, taken for a typical elliptical galaxy at $z=0$ from Fukugita et al. (1995).

The SG analysis of this catalogue shows the existence of a gravitationally bound structure of 27 galaxies (Fig. 10). The velocity histogram of these 27 galaxies is displayed in Fig. 11 (bottom), together with the velocity histogram of the full sample of 49 galaxies (top). The biweight velocity dispersion of these 27 galaxies is $1968 \mathrm{~km} \mathrm{~s}^{-1}$, a value notably higher than the overall cluster velocity dispersion of $\sigma_{\mathrm{v}}=758 \mathrm{~km} \mathrm{~s}^{-1}$ estimated in Sect. 1 and suggesting that substructuring remains within these 27 galaxies, as also suggested by the velocity histogram of Fig. 11. It is likely that out of these 27 galaxies, 10 are probably foreground objects. The mass of $4.4 \times 10^{13} M_{\odot}$ estimated for the 27-galaxy structure is therefore most probably an overestimate. If we exclude the 10 foreground objects and consider only the 17 galaxies that are likely to be gravitationally bound, their velocity dispersion is then $583 \mathrm{~km} \mathrm{~s}^{-1}$.

A more strongly gravitationally bound structure of 14 galaxies is found within this structure of 27 galaxies. It has a mass of $1.5 \times 10^{13} M_{\odot}$ and a velocity dispersion of $875 \mathrm{~km} \mathrm{~s}^{-1}$. Its velocity histogram is shown in blue in Fig. 11 (bottom) and suggests that 2 galaxies are foreground objects; if these 2 galaxies are excluded, the velocity dispersion becomes $640 \mathrm{~km} \mathrm{~s}^{-1}$.

If we now consider all the galaxies within a radius of $5 \mathrm{Mpc}$ with measured redshifts with or without measured magnitudes, we have a list of 86 galaxies. Out of these, 43 galaxies have velocities between 14700 and $18000 \mathrm{~km} \mathrm{~s}^{-1}$, corresponding to 

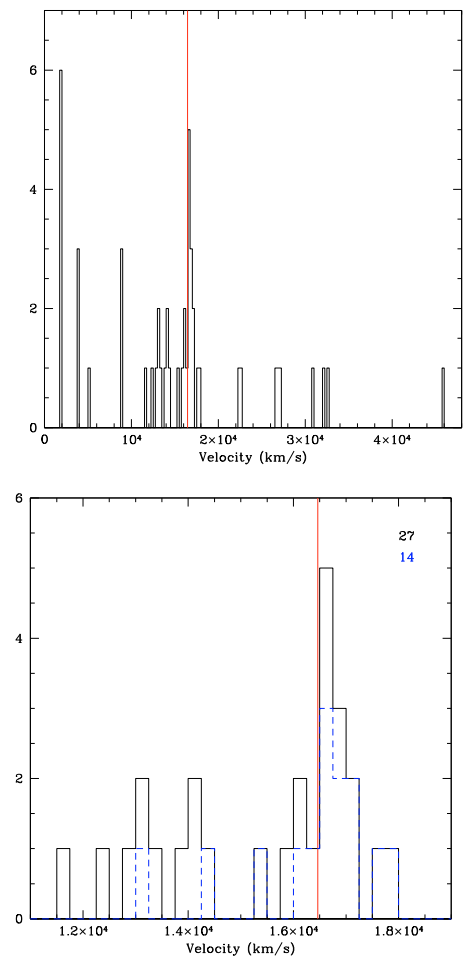

Fig. 11. Top: velocity histogram of the 49 galaxies with measured redshifts and magnitudes in a $5 \mathrm{Mpc}$ circle around Abell 780. Bottom: velocity histograms of the 27 (in black) and 14 (in blue) galaxies found to be gravitationally bound by the SG method. The red vertical line indicates the velocity of the $\mathrm{cD}$ galaxy of Abell 780 .

the cluster velocity interval, and the velocity dispersion of these 43 galaxies is $\sigma_{\mathrm{v}}=741 \mathrm{~km} \mathrm{~s}^{-1}$, almost exactly what is derived from X-rays.

In view of the optical data available for Abell 780, this cluster appears to have an overall relaxed nature, with no or hardly any infall of field dwarf/faint galaxies. This agrees with the large scale X-ray properties of Abell 780 derived from XMM-Newton data by Simionescu et al. (2009a,b). However, deeper and broader field imaging and more galaxy redshifts are obviously necessary to achieve a better understanding of the Abell 780 cluster, particularly on small scales where Chandra $\mathrm{X}$-ray data have revealed a wealth of structures (Wise et al. 2007).

Acknowledgements. We warmly thank the referee for many constructive comments that helped improve the paper. We acknowledge help from G. Mars and G. Daste for part of the data reduction. We are very grateful to G. Boué for making several pieces of his software available to us and for helping us use them. We also thank Brian McNamara for sending us the ds9 region file showing the positions of the X-ray cavities.

\section{References}

Adami, C., Picat, J.-P., Savine, C., et al. 2006a, A\&A, 451, 1159 Adami, C., Scheidegger, R., Ulmer, M., et al. 2006b, A\&A, 459, 679

Adami, C., Durret, F., Mazure, A., et al. 2007a, A\&A, 462, 411

Adami, C., Picat, J. P., Durret, F., et al. 2007b, A\&A, 472, 749

Andreon, S., Puddu, E., de Propris, R., et al. 2008, MNRAS, 385, 979

Batuski, D. J., Burns, J. O., Newberry, M. V., et al. 1991, AJ, 101, 1983

Beijersbergen, M., Hoekstra, H., van Dokkum, P. G., et al. 2002, MNRAS, 329, 385

Bertin, E., \& Arnouts, S. 1996, A\&AS, 117, 393

Boué, G., Adami, C., Durret, F., Mamon, G., \& Cayatte, V. 2008, A\&A, 479, 335

Burns, J. O., Rhee, G., Owen, F. N., et al. 1994, ApJ, 423, 94

Carlberg, R. G., Yee, H. K. C., \& Ellingson, E. 1997, ApJ, 478, 462

David, L. P., Arnaud, K. A., \& Forman, W. 1990, ApJ, 356, 32

Fasano, G., Marmo, C., Varela, J., et al. 2006, A\&A, 445, 805

Fukugita, M., Shimasaku, K., \& Ichikawa, T. 1995, PASP, 107, 945

Girardi, M., Fadda, D., Giuricin, G., et al. 1996, ApJ, 457, 61

Lobo, C., Biviano, A., Durret, F., et al. 1997, A\&A, 317, 385

McCracken, H. J., Radovich, M., Bertin, E., et al. 2003, A\&A, 410, 17

McNamara, B. R., Wise, M., Nulsen, P. E. J., et al. 2000, ApJ, 534, L135

Mohr, J. J., Mathiesen, B., \& Evrard, A. E. 1999, ApJ, 517, 627

Owen, F. N., White, R. A., \& Burns, J. O. 1992, ApJS, 80, 501

Ramella, M., Biviano, A., Pisani, A., et al. 2007, A\&A, 470, 39

Robin, A. C., Reylé, C., Derrière, S., et al. 2003, A\&A, 409, 523

Schlegel, D. J., Finkbeiner, D. P., \& Davis, M. 1998, ApJ, 500, 525

Serna, A., \& Gerbal, D. 1996, A\&A, 309, 65

Simionescu, A., Werner, N., Böhringer, H., et al. 2009a, A\&A, 493, 409

Simionescu, A., Roediger, E., Nulsen, P. E. J., et al. 2009b, A\&A, 495, 721

Wise, M. W., McNamara, B. R., Nulsen, P. E. J., Houck, J. C., \& David, L. P. 2007, ApJ, 659, 1153 